\title{
La educación como política pública en la democracia: una perspectiva hermenéutica en el siglo XXI
}

\section{Education as a public policy in democracy: A hermeneutical perspective in the 21 st century.}

\author{
Maroslee Díaz Guillén \\ Magíster en Gerencia Educacional \\ Universidad Pedagógica Experimental Libertador, Venezuela \\ Marosleediaz@gmail.com n https://orcid.org/0000-0002-2139-4163 \\ Emgelberth Vargas Monzón \\ Doctor en Ciencias de la Educación \\ Universidad Bicentenaria de Aragua, Venezuela \\ Evargas@gmail.com.do n https://orcid.org/0000-0003-3098-0719
}

Fecha de recepción: 20 de octubre de 2020

Fecha de aceptación: 13 de noviembre de 2020

Fecha de publicación: 1 de enero de 2021

Favor citar este artículo de la siguiente forma:

Díaz Guillén, M. y Vargas Monzón, E. (2021). La educación como política pública

en la democracia: Una perspectiva hermenéutica en el siglo XXI

AULA Revista de Humanidades y Ciencias Sociales, 67 (1), 43-50

$\mathrm{https}: / /$ doi.org/10.33413/aulahes.2021.67i1.152

\section{RESUMEN}

La educación en el siglo XXI es vista como la piedra medular de la sociedad, la cual se cumple como parte de las políticas públicas de Estado en un sistema de gobierno democrático. En esta investigación se busca indagar sobre la noción que se posee sobre democracia en el siglo XXI para poder describir la relación existente entre la democracia y el cumplimiento de las políticas públicas según otros investigadores. El eje teleológico central es generar una perspectiva hermenéutica sobre la educación como política pública de la democracia en el siglo XXI. El fundamento metodológico se llevó a cabo desde el paradigma postpositivista con enfoque cualitativo, bajo la técnica de investigación documental y un procesamiento de la información mediante el análisis de contenido. Las reflexiones que surgieron a partir de la perspectiva hermenéutica del discurso de otros actores sociales, permitieron develar que la educación es un derecho humano esencial para el desarrollo sostenible de los países, que además se considera una política pública que garantiza el sentido de democracia.

Palabras clave: democracia, educación, políticas públicas.

\begin{abstract}
Education in the XXI century is seen as the cornerstone of society which is fulfilled as part of the public policies of the State in a democratic government system. This research seeks to investigate the notion of democracy in the 21 st century in order to describe the existing relationship between democracy and compliance with public policies according to other researchers. Likewise,
\end{abstract}


the importance of education as public policy in the 21 st century will be understood. The central teleological axis is to generate a hermeneutical perspective on education as a public policy of democracy in the XXI century. The methodological foundation was carried out from the postpositivist paradigm with a qualitative approach under the documentary research technique and information processing through content analysis. The reflections that arose from the hermeneutical perspective of the discourse of other social actors allowed to reveal that education is an essential human right for the sustainable development of the countries, it is also considered a public policy that guarantees the sense of democracy.

Keywords: democracy, education, public policies.

\section{Introducción}

«La educación es considerada como la acción de educar, formar, capacitar, alfabetizar, y generar la construcción de conocimientos en el educando; pero también es considerada como un derecho universal que se centra en la educación que todo ser humano debe poseer en sus bases más fundamentales». Es así como lo señala Freire (1997. p.104).

La raíz más profunda de la politicidad de la educación está en la propia educabilidad del ser humano, que se funde en su naturaleza inacabada y de la cual se volvió inconsciente. Inacabado y consciente de inacabamiento histórico, el ser humano se haría necesariamente un ser ético, un ser de opción, de decisión.

A partir de esta concepción, esa necesidad del ser humano de ser educado para así poder tener el derecho inmutable a la participación ciudadana, es propia de los sistemas de gobierno democrático. La Organización de las Naciones Unidas (ONU, 1948) en la Declaración Mundial de los Derechos Humanos, incita a las naciones al cumplimiento del derecho a la educación como una política de Estado. En esta investigación de tipo documental, se busca generar una perspectiva hermenéutica de la educación como política pública de la democracia en el siglo XXI. Se parte de la necesidad académica de abordar diversas nociones de otros investigadores en relación a estas categorías, para dar a conocer a los ciudadanos de la República Dominicana su derecho innegable a la educación. Desde el punto de vista investigativo, permite la interpretación de la información abordada mediante una postura postpositivista y un enfoque cualitativo. Desde el punto de vista social, genera una episteme global sobre esta tríada: educación-política pública-democracia.

\section{Develando realidades}

«La educación

no solo enriquece la cultura...

Es la primera condición para la libertad, la democracia y el desarrollo sostenible».

Kofi Annan.

La educación es la piedra medular de todas las naciones convirtiéndose en la base del desarrollo sostenible de las mismas. Se establece como un derecho según la ONU (1948), en la Declaración Mundial de los Derechos Humanos, donde sostiene que toda persona debe ejercerlo de manera pública, gratuita y obligatoria en su estado elemental y fundamental de instrucción; mientras que la técnica y profesional deberá ser generalizada. Asimismo, se debe tomar en cuenta que esta debe enfocarse en el desarrollo de la personalidad humana y el respeto al cumplimiento de los derechos humanos, lo que permitirá fortalecer los nexos entre naciones así como la tolerancia e inclusión de la diversidad cultural existente en pro de la paz.

En este sentido, la educación parte de ser un derecho fundamental de todo ser humano para convertirse en una política de Estado, y se considera entonces entre las políticas públicas, 
pudiendo definirse estas según Cejudo y Michel como: «un conjunto de acciones específicas orientadas a resolver un problema público acotado o concreto» (p.4). Esto quiere decir que, si la educación se toma en cuenta como política pública, es para solventar el problema que subyace cuando las personas no son alfabetizadas, formadas y capacitadas en una nación. Poder dar cumplimiento al derecho a la educación en la población permitirá el progreso sostenible en todas las dimensiones que establece la Organización de las Naciones Unidas para la Educación, la Ciencia y la Cultura (UNESCO, 2010) en la Lente de la Educación para el Desarrollo Sostenible, entre las que se menciona la dimensión de sociedad, medio ambiente, economía y cultura.

Ahora bien, esta sostenibilidad que deben llevar a cabo las naciones tiende a estar relacionada con el derecho al ejercicio de la soberanía, el cumplimiento de las políticas que beneficien a la sociedad, que mejoren las condiciones y cosmovisión que se posea sobre el medio ambiente; y que también mejore la economía y el tratamiento que se le da en pro de un futuro y una mejor calidad de vida, así como un cambio cultural que invite al reconocimiento de los deberes y derechos humanos que posee cada persona.

Por otro lado, poseer una perspectiva hermenéutica de la educación como política pública en la democracia implica la revisión de diversas concepciones. Es así como lo plantea Kant, citado por Deval (1993), «el hombre puede considerar como los dos descubrimientos más difíciles el arte de gobierno y el de la educación y, sin embargo, se discute aún sobre estas ideas» (p.1). Esta relación ha sido objeto de estudio por innumerables investigadores, no obstante, se debe tener claro que el ejercicio de los derechos de una persona a ser educada surge en la democracia. Incluso desde la edad antigua, el impartir la educación se correspondía con una política pública, es así como lo hacen saber algunos clásicos literarios como La República de
Platón o La Política de Aristóteles. Desde entonces, el ejercicio de la democracia ha estado inmerso en el derecho a la educación como parte de la política pública.

Partiendo de esta postura surgen las siguientes interrogantes:

¿Cuál es la noción que se posee sobre democracia en el siglo XXI?

¿Cómo se la relaciona la democracia con el cumplimiento de las políticas públicas según otros investigadores?

¿Cuál es la importancia de la educación como política pública en el siglo XXI?

\section{Ejes teleológicos}

-Eje central

- Generar una perspectiva hermenéutica sobre la educación como política pública de la democracia en el siglo XXI.

-Ejes específicos

- Indagar respecto a la noción que se posee sobre democracia en el siglo XXI.

- Describir la relación existente entre democracia y el cumplimiento de las políticas públicas según otros investigadores.

- Comprender la importancia de la educación como política pública en el siglo XXI.

\section{Fundamentos epistemológicos}

«La democracia no puede tener éxito a menos que, a los que expresan su elección, se les prepare para elegir sabiamente.

La verdadera salvaguardia de la democracia, por lo tanto, es la educación». Franklin Roosevelt.

\section{Democracia en el siglo XXI}

El término democracia, según la Real Academia Española (2020), proviene etimológicamente del latín tardío democratia y este a su vez proviene del griego démokratía que significa gobierno donde las políticas son ejercidas por 
los ciudadanos. Del mismo modo es vista como una doctrina donde la soberanía reside en el pueblo con igualdad de derechos individuales, donde existe la participación y el respeto a la diversidad cultural. Es entonces la democracia la forma de gobierno en la que el énfasis está en beneficiar a la sociedad en el cumplimiento de sus deberes y derechos, cubrir sus necesidades y procurar mejorar su calidad de vida en las bases de la libertad, el respeto, la igualdad y la soberanía.

Entre las diversas concepciones que poseen investigadores del siglo XXI sobre la democracia, se tiene la señalada por Sermeño y Aragón (2017) al decir que: «existen distintas maneras de manejar la relación entre la teoría y la práctica de la democracia, entre el ideal y sus realizaciones» (p.5). Estos autores hacen mención de la democracia como aquellas políticas que se establecen, pero que están lejos de cumplirse por las acciones de los gobernantes... Esta problemática incide de manera directa en la sociedad, ya que la falta de atención a las necesidades que se presentan en la sociedad, pone en riesgo su calidad de vida.

Por otro lado, Monsiváis (2013) señala que: «Para fortalecer la democracia, y en especial las democracias emergentes, hacen falta visión y voluntad políticas, pero también la capacidad de resolver cuestiones prácticas de diseño institucional y edificación de programas» (p.26). Este autor no considera despolitizar a la democracia, sino hacer una introspección sobre su ontología política, una realidad que permitirá develar el status de esta en función del cumplimiento de las teorías en la práctica, pues la sostenibilidad de la democracia va a depender de la consecución de las políticas públicas.

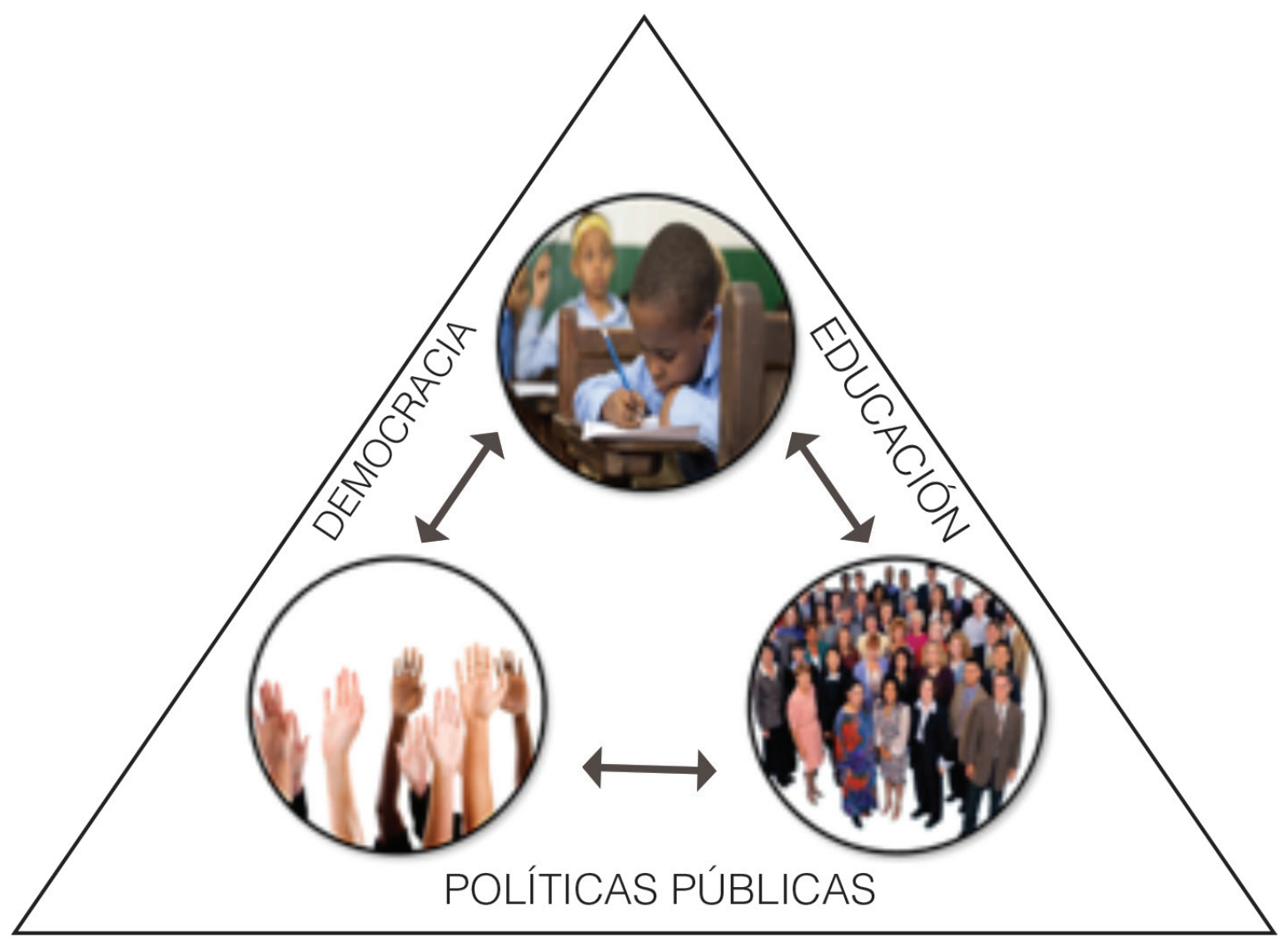

Figura 1. Democracia en el siglo XXI. 
Para Medina (2015), la democracia se refleja en la educación, que es la que permite las mejoras en la participación ciudadana. Este autor señala:

Una de las funciones principales de la educación es contribuir al desarrollo del carácter de los individuos con la sociedad, de tal forma que a través del sistema educativo se ponga en marcha la idea de «educar para la libertad y en la libertad» (p.110). De ahí que, en la tarea de cultivar el carácter moral, educación, libertad y democracia hayan de ser interdependientes.

Esta interdependencia a la que hace mención el autor es característica de la democracia. En efecto, una democracia sin libertad no puede llamarse democracia, una democracia donde la sociedad no posea el derecho a la alfabetización y la toma de decisiones políticas, no puede llamarse democracia.

\section{La democracia y las políticas públicas}

Una vez descrita la democracia, es necesario establecer su relación con las políticas públicas, pues es notorio que para que las políticas se lleven a cabo de manera satisfactoria debe existir una participación por parte de la sociedad. De ocurrir todo lo contrario, los resultados positivos se irán reduciendo significativamente, es así como lo plantean Martínez, Díez, Rogero, Anguita y Rivas (2017), al sostener que:

La reducción de la participación y de la capacidad de decisión sobre la política educativa a las estructuras e instituciones de los partidos políticos en el marco de la democracia formal, empobrece y recorta el sentido original y la fuerza real de la democracia (p.40).

Estos autores señalan que esta reducción participativa conlleva a la confusión de los ciudadanos con el ejercicio de las políticas, pues lo lógico es que la teoría se cumpla en la práctica; pero si se existe una reducción como la mencionada, tiende a desarrollarse un condicionamiento sobre las acciones democráticas. Para estos autores el pensamiento sobre la política posee una relación estrecha con «... espacio público común, dialógico y plural» ( $\mathrm{p} .40)$. Esta posición hace referencia del espacio público común como aquel que debe existir en democracia respecto a la integración de la ciudadanía en las decisiones que comprometen sus derechos, deberes y vida social.

Es relevante mencionar la concepción que sostienen Cruz y Mballa (2017) al referirse a la necesidad de que los mecanismos de participación ciudadana no se conviertan en instrumentos del aparato político del poder ejecutivo, pues se hace perentorio que este se mantenga como espacio en donde emerja un ambiente de igualdad, inclusión social y por tanto, la construcción de la ciudadanía. En el mismo orden de ideas, estos autores establecen que la participación ciudadana debe ser dotada de autonomía para que se establezca una cultura cívica que permita la libertad de expresión y la invitación a la reflexión y construcción de opiniones propias.

La democracia y las políticas públicas son unas variables indisolubles que conforman un sistema o forma de gobierno, pero a su vez son variables que se engranan a otras también esenciales como la participación ciudadana, la libertad de pensamiento, el derecho al ejercicio electoral o político y la inclusión, entre otros. Se hace evidente que, al existir un reduccionismo en las participaciones de los ciudadanos, las políticas pierden la esencia del sentido democrático.

\section{La educación como política pública en el siglo XXI}

La educación como política pública en pleno siglo XXI es más que una necesidad. El crecimiento poblacional de las naciones implica cada vez más esfuerzo por llevar a cabo el cumplimiento del derecho a una educación libre, gratuita y obligatoria en sus bases fundamentales; no obstante, no se excluye la posibilidad de que las personas quieran acceder a la educación privada en una sociedad que se muestra 
libre y democrática. Con relación a lo expuesto, Pulido (2017) señala lo siguiente:

La educación pública constituye el ámbito por excelencia de realización del derecho a la educación... cualquier intento por debilitarla debe ser considerado como un ataque directo a su disfrute y realización. No excluye la posibilidad de opción por la educación privada para quienes tengan los medios para realizar allí este derecho, pero define taxativamente el papel regulador del Estado y su indeclinable responsabilidad en esta materia a través de la educación pública (p.24).

Como puede evidenciarse, el compromiso democrático del Estado en hacer realidad el derecho de las personas a la educación, no se exime la creación de instituciones del sector privado, todo lo contrario. El Estado tiene el deber de ofrecer calidad educativa tanto para los discentes como para los docentes. Cabe destacar que en pleno siglo XXI con el auge de la revolución tecnológica, las necesidades educativas de la población han conllevado la creación de políticas públicas relacionadas con el ámbito educativo, para cubrir las necesidades de la sociedad, no obstante, se tiende a no llevar a la práctica muchas de estas políticas.

\section{Bases legales}

En República Dominicana, el derecho a la educación es un deber establecido en la Constitución (2010), en su Artículo 63, que reza lo siguiente: "Toda persona tiene derecho a una educación integral, de calidad, permanente, en igualdad de condiciones y oportunidades, sin más limitaciones que las derivadas de sus aptitudes, vocación y aspiraciones». Como puede evidenciarse, se declara el cumplimiento al derecho a la educación que se establece como un derecho universal de la humanidad.

En el mismo orden de ideas, mediante la Ley General de Educación N ${ }^{\circ}$ 66-97 aprobada en 1997 en su Artículo 1, se garantiza el derecho de todo el país a la educación como labor del Estado y de los organismos pertinentes.

\section{Fundamentos metodológicos}
«Mi ideal político es el democrático. Todo el mundo debe ser respetado como persona y nadie debe ser divinizado».
Albert Einstein.

Entre los fundamentos metodológicos que sustentan esta investigación se debe tomar en cuenta que la misma se ubica en un paradigma postpositivista, reconociéndose así la postura de Kunt (1962) al sostener como paradigma el modo en cómo se percibe el mundo, mientras que el paradigma postpositivista se refiere según Martínez (2002) a: «el rescate del sujeto y de su importancia» (p.38). De este modo, la observación que caracteriza al paradigma positivista no sería utilizada solo desde lo empírico, sino desde la visión de los valores, intereses, actitudes y creencias. De allí surge la frase de Polanyi citado por Martínez (2002), «todo conocimiento es conocimiento personal» (p.38).

\section{Enfoque y tipo de investigación}

El enfoque de esta investigación es cualitativo, debido a que busca interpretar las nociones de otros investigadores basándose en la subjetividad. Al respecto Gómez (2006), señala que este se refiere a: «métodos de recolección de datos sin medición numérico, sin conteo. Utiliza las descripciones y las observaciones» (p.60). Gómez se refiere al implemento de la obtención de la información a partir del estudio del fenómeno y de los sujetos que se encuentran inmersos en él; de la misma manera que el tipo de investigación usada es la descriptiva.

\section{Técnicas de la investigación}

Este estudio se llevó a cabo bajo la investigación documental conocida por Tena y Rivas 
(1995) como: «aquella que se encuentra contenida en diversas observaciones o datos, contenidas en escritos de diversos tipos» (p.49). Tal como lo señalan los autores, una investigación documental comprende las observaciones y escritos de otros investigadores en relación al temático objeto de estudio. De la misma forma, se hace conocer que el procesamiento de la información se realizó mediante un análisis de contenido partiendo de las categorías democracia, políticas públicas y educación.

\section{Reflexiones}

«La democracia tiene responsabilidades profundas con aquellos que habitan en ella. Y su mayor responsabilidad es, por supuesto, la educación, la distribución del ingreso, que nadie pase hambre, que nadie sea un analfabeto, y posibilidades de trabajo para todos. Si la democracia no puede dar esto... Y bueno, está fallando algo...» Jean Paul Sartre.

Durante el recorrido documental de las categorías de esta investigación se pudo comprender la

\section{Referencias}

Cejudo, G., y Michel, C. (2016). Coherencia y políticas públicas. Metas, instrumentos y poblaciones objetivo. Gestión y Política Pública. 25, ( 1). http://www.scielo.org. $\mathrm{mx} / \mathrm{pdf} / \mathrm{gpp} / \mathrm{v} 25 \mathrm{n} 1 / \mathrm{v} 25 \mathrm{n} 1 \mathrm{a} 1 . \mathrm{pdf}$

Constitución de la República Dominicana. (2010). Gaceta Oficial. $\mathrm{N}^{\mathrm{o}}$ 105611. http://dominicana.gob.do/index. php/pais/2014-12-16-20-52-13

Cruz, L., y Mballa, L. (2017). Mecanismos de participación ciudadana en las políticas públicas en América Latina. Revista Políticas Públicas. 10, (1). http://www.revistas. usach.cl/ojs/index.php/politicas/article/view/2963/2706

Freire, P. (1997). La Pedagogía de la Autonomía. Siglo XXI editores.

Gómez, M. (2006). Introducción a la metodología de la investigación cientifica. Brujas.

Kunt, T. (1978). La estructura de las revoluciones cientificas. FCE.

Ley 66-97. Ley General de Educación. República Dominicana. importancia de la educación como política pública en pleno siglo XXI, dando a conocer que la educación es esencial para el desarrollo de los países; asimismo es un derecho humano que permite la construcción de pensamientos propios en los ciudadanos. Se considera una política pública que al ser cumplida garantiza el sentido de democracia. Un país donde se cumple el derecho universal a ser educado e instruido desde sus bases fundamentales, tiene la garantía de progreso dentro de las dimensiones en las que se desenvuelve la sociedad.

La UNESCO (2010) hace énfasis en la sostenibilidad que deben poseer las naciones y para que esta se cumpla se hace necesaria la democracia, y por consiguiente la educación. Esta investigación permitió generar esta perspectiva hermenéutica partiendo de la interpretación de las informaciones recabadas por otros investigadores; lo que ha llevado a reflexionar sobre la importancia y el interés que posee la comunidad científica sobre el desarrollo de las naciones, la diversidad de las concepciones democráticas y la necesidad del cumplimiento de las políticas públicas por parte del Estado.

https://www.oas.org/juridico/spanish/mesicic2_repdom_ sc_anexo_7_sp.pdf

Martínez, M. (2002). La nueva ciencia. Su desafío, lógica y método. Editorial Trillas.

Martínez, J., Díez, E., Rogero, J., et al. (2017). La democracia, sus agentes, sus agendas, cuando se construyen las políticas públicas de educación. Revista Educación, Politica y Sociedad. 2, (2). https://repositorio.uam.es/ bitstream/handle/10486/679704/REPS_2_2_3.pdf?sequence $=1 \&$ is Allowed $=\mathrm{y}$

Medina, J. (2015). Educación democrática y política educativa: El sistema educativo a debate. Cuestiones Pedagógicas, 24. Universidad de Sevilla. https://institucional.us.es/ revistas/cuestiones/24/08_24.pdf

Monsiváis, A. (2013). La democracia como política pública: Oportunidades para el fortalecimiento democrático. Revista de Estudios Sociales, 47. https://journals.openedition.org/ revestudsoc/7962\#tocto1n1 
Organización de las Naciones Unidas. (1948). Declaración Mundial de los Derechos Humanos. https://www. un.org/es/universal-declaration-human-rights/

Organización de las Naciones Unidas para la Educación, la Ciencia y la Cultura. (2010). La Lente de la Educación para el Desarrollo Sostenible: Una herramienta para examinar las políticas y la práctica. La educación para el desarrollo sostenible en acción. Instrumentos de aprendizaje y formación. https://www.miteco.gob. es/es/ceneam/recursos/pag-web/lente-educacion-dllosostenible.aspx

Pulido, O. (2017). Política pública y política educativa: Una reflexión sobre el contexto. Educación y
Ciudad, 33. https://dialnet.unirioja.es/descarga/articulo/6213573.pdf

Real Academia Española. (2019). Diccionario de la Lengua Española. Asociación de Academias de la Lengua Española. https://dle.rae.es

Sermeño, A., y Aragón, A. (2017). La democracia en el siglo XXI. La reinvención de la representación política. Entrevista a Andrea Greppi. Revista Andamios, 14, (35). http://www.scielo.org.mx/pdf/anda/v14n35/18700063-anda-14-35-00213.pdf

Tena, A., y Rivas, R. (2007). Manual de investigación documental. Elaboración de tesinas (4a ed.). Plaza y Valdés.
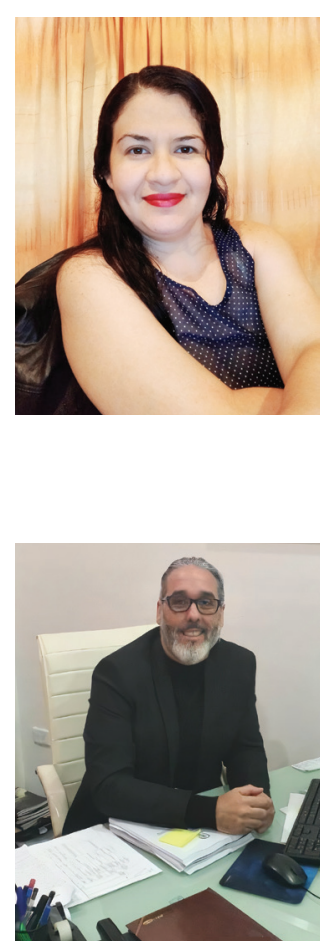

\section{Maroslee Díaz Guillén}

$\mathrm{PhD}$ (c) profesora de Biología y Química (UPEL). Especialista en Tecnologías de la Información y Comunicación en la Educación (UNESR), Magíster en Gerencia Educacional (UPEL), certificada como Metodólogo de la Investigación (UPEL). Actualmente está cursando estudios de Doctorado en Ciencias de la Educación(UPEL). Tiene una Maestría en Educación a Distancia (UNA), Diplomado en Investigación (UPEL), Estadística para la Investigación en las Ciencias Sociales (ULA) y en Filosofia (UC), y es experta en Educación Virtual (FATLA). Docente universitaria de postgrado. Con formación académica constante, apuesta a la investigación como base primordial en el desarrollo sostenible de los países.

\section{Emgelberth Vargas}

Ingeniero Geofísico, de La Universidad Central de Venezuela; Máster en Gerencia de Proyectos y en Especialización en Cambio Climático y Doctor en Ciencias de la Educación por la Universidad Bicentenaria de Aragua, Venezuela. Labora en diferentes ramas del ámbito académico e investigación, con funciones de dirección, coordinación, apoyo académico, docencia e investigación, tanto en grado como en postgrado. Actualmente cursa el PhD en Física en la Universidad Central de Venezuela. 